\title{
Evaluación del desempeño y análisis de eficiencia del nivel sigma en la evaluación de la calidad del servicio en una institución de educación superior
}

\author{
Tomás J. Fontalvo ${ }^{1^{*}}$, Enrique J. De la Hoz y ${ }^{2}$ Nubia Marrugo ${ }^{3}$ \\ (1) Facultad de Ciencias Económicas, Programa de Administración, Universidad de Cartagena, Campus piedra de \\ Bolívar, Cartagena - Colombia. (correo-e: tfontalvoh@unicartagena.edu.co) \\ (2) Facultad de Ingeniería, Programa Ingeniería Industrial, Universidad Tecnológica de Bolívar, Campus de Ternera, \\ Cartagena - Colombia. (correo-e: edelahoz@utb.edu.co) \\ (3) Facultad de Derecho, Programa de derecho, Universidad libre, Barranquilla - Colombia. \\ (correo-e: nmarrugo@unilibrebaq.edu.co)
}

${ }^{*}$ Autor a quien debe ser dirigida la correspondencia

Recibido Abr. 9, 2020; Aceptado Jun. 5, 2020; Versión final Ago. 26, 2020, Publicado Dic. 2020

\begin{abstract}
Resumen
Esta investigación presenta un método para evaluar el desempeño y analizar la eficiencia del nivel sigma de las dimensiones de calidad del servicio en una institución de educación superior. Como concepción teórica se articulan los conceptos de calidad del servicio, las métricas de Seis Sigma y el análisis envolvente de datos. El tipo de investigación fue evaluativa, considerando como información primaria de entrada los registros recibidos por el centro de gerencia del servicio durante 11 meses en el año 2019. Los resultados muestran que el nivel de eficiencia promedio del servicio fue del $93,48 \%$ en los 11 meses evaluados. Se identificaron las dimensiones de calidad de entrada críticas para mejorar la satisfacción de los clientes. Se concluye que el método de evaluación del desempeño y análisis de eficiencia del nivel sigma en la calidad del servicio permite valorar las dimensiones de calidad del servicio de forma puntual, periódica y longitudinalmente.
\end{abstract}

\section{Performance evaluation and efficiency analysis of the sigma level in the evaluation of service quality at a higher education institution}

\begin{abstract}
This research study presents a method to assess the sigma level performance and efficiency of quality dimensions at a higher education institution. A theoretical context is provided on the quality of the service, including the Six Sigma metrics and the data envelopment analysis. Data input are records received by the service management center for 11 months in 2019. The results show that the average service level efficiency was $93.48 \%$ during the 11 months examined. Critical input quality dimensions are identified to improve customer satisfaction. It is concluded that the performance evaluation method and the sigma quality service efficiency analysis performed here allow assessing quality services dimensions accurately, periodically, and longitudinally.
\end{abstract}

Keywords: service quality; Six Sigma; education; control charts 


\section{INTRODUCCIÓN}

Hoy en día los grupos de interés son determinantes para el desarrollo de una organización, y más las instituciones educativas que garantizan el desarrollo de un país y una sociedad. Sin embargo, en estos contextos las condiciones de globalización han generado clientes cada vez más exigentes. Lo cual requiere el uso de técnicas y herramientas que no solo midan la calidad de forma cualitativa, sino que se apoyen en herramientas de estadística avanzada y en la optimización de las variables asociadas para la evaluación de la calidad y la eficiencia del servicio. Para afrontar las necesidades de los clientes las instituciones de educación superior poseen centros de gerencia del servicio con el fin de captar, analizar, procesar y tomar acciones que contribuyan con el monitoreo de la calidad de forma particular y de manera integral. Desde esta perspectiva en esta investigación se hizo necesario, establecer como evaluar el desempeño de las dimensiones de calidad de manera integral, apoyadas en herramientas avanzadas de estadística. De igual manera se buscó analizar el desempeño de forma puntual, periódica, longitudinalmente; así como también analizar la eficiencia global y holística en múltiples periodos, de tal manera que se puedan identificar los periodos de excelencia y los periodos críticos. Así mismo, se busca analizar cómo se alcanza la eficiencia del desempeño de las dimensiones de calidad por periodos y la eficiencia global. Es importante señalar que diferentes investigaciones previas (Fontalvo et al., 2019: Fontalvo et al., 2018) muestran la pertinencia de utilizar métodos de doble fase para evaluar organizaciones, lo que facilita la toma de decisiones objetivas y el mejoramiento continuo.

Todo lo planteado previamente, nos llevan a generar las siguientes preguntas problemas de esta investigación ¿Cómo valorar el nivel de desempeño sigma de las dimensiones de calidad del centro de gerencia del servicio en una institución de educación superior? ¿Cómo establecer una estructura de evaluación de la eficiencia técnica de la calidad del servicio en una institución de educación superior? ¿Cómo estructurar el método de valoración de la eficiencia para medir los desempeños de los niveles sigma de las dimensiones de calidad de un centro de gerencia de servicio en una institución de educación superior? ¿Cuáles son los niveles de eficiencias de los desempeños del nivel sigma de las dimensiones de calidad en cada periodo del año y de manera global en la institución objeto de estudio? El análisis previo, genera como objetivos de esta investigación proponer un método para evaluar el desempeño y realizar un análisis de la eficiencia técnica del nivel sigma de las dimensiones de calidad del servicio en una institución de educación superior de manera integral, objetiva y periódica. De tal manera, que se tengan diferentes criterios para monitorear las dimensiones de calidad en un centro de gerencia de servicio en una institución de educación superior.

La preguntas problemas generadas en esta investigación nos llevan a los siguientes objetivos i) establecer un estructura para valorar la eficiencia técnica de las dimensiones de calidad del servicio de la institución objeto de estudio ii) Valorar y analizar el nivel de desempeño del nivel sigma de las dimensiones de calidad del servicio iii) establecer la estructura de medición de Análisis Evolvente de Datos DEA que permite evaluar el nivel de eficiencia del nivel sigma de la dimensión de calidad de salida propuesta, de manera periódica y de forma global del centro de gerencia del servicio iv) Valorar y analizar el nivel de eficiencia de manera periódico y de forma global de los niveles de desempeño del nivel sigma de las dimensiones de calidad del centro de gerencia del servicio v) Proponer la Matriz de proyección de desempeño del nivel sigma de calidad requerido de las dimensiones de calidad de entrada, para identificar las dimensiones que requieren mejoras con el fin de lograr la eficiencia de la dimensión de calidad de salida

Diferentes autores señalan, cómo el sector de los servicios ha venido asumiendo las concepciones y prácticas del sector productivo, adaptando estas particularidades al servicio (Tumino y Poitevin, 2014). Considerando la importancia de los servicios educativos, se ha generado un movimiento para usar herramientas y planteamientos para la mejora de estos (Boon, 2016). En otras investigaciones los autores (Yildiz y Kara, 2015) afirman que, aunque las entidades educativas no compiten con la voracidad de otras, las entidades que no asuman nuevas concepciones y modelos de calidad no podrán competir y subsistir en el futuro. De igual manera otros investigadores (Fontalvo y De la hoz, 2018) mostraron la pertinencia de utilizar estándares y herramientas de calidad asociados a los sistemas de calidad para mejorar la calidad de las instituciones de educación superior. Este mismo enfoque es desarrollado por los investigadores (Capaldo, Costantino y Pellegrino, 2017) dándole importancia a la normalización y herramientas de gestión de la calidad. Por otro lado, los investigadores Javed y llyas (2018) analizan y plantean la importancia de analizar diversos constructos de calidad con el fin de cumplir con las necesidades y expectativas de los grupos de interés. Otros investigadores han estudiado y analizado el comportamiento del uso de otro tipo de indicadores y estándares para mejorar la productividad de las organizaciones (Fontalvo, 2014) y han demostrado la pertinencia para monitorear el desempeño de diversas organizaciones. En este mismo sentido otros estudios recalcan que la mejor manera de generar competitividad en el contexto donde se interviene es prestando un servicio de alta calidad, para lo cual es necesario herramientas de control de calidad efectivas (Fazil y Muzamil, 2019) 
Anthony y Antony (2017) compararon universidades del Reino unido y del resto mundo y mostraron como Seis sigma es pertinente para mejorar los procesos administrativos, sin embargo la metodología de seis sigma, no tienen el mismo impacto a nivel de investigación y docencia. Por otra parte Jiju (2017) plantea la necesidad de hacer asequible los procesos estadísticos relacionados con las métricas de Seis sigma con el fin de impactar positivamente en la instituciones de educación superior. Otros estudios (Subbarayalu y Al Kuwaiti, 2017) aplicaron Seis sigma y sus métricas en una facultad académica, para lo cual fue necesario involucrar a estudiantes, profesores con el fin de aplicar el ciclo Definir, Medir, Analizar, Mejorar y Controlar (DMAIC) a los diferentes procesos. Estudios muestran la importancia de establecer niveles de desempeño en las dimensiones de calidad de los servicios que prestan las entidades de servicios (Shokri, 2013). Hoy en día el método de Seis sigma se viene implementado en diferente contextos, especialmente por su versatilidad y su utilidad para establecer unas métricas puntuales que se pueden valorar fácilmente y a la postre traducirse en mejoras que pueden cuantificarse y valorarse, lo que es pertinente para los diferentes grupos de interés en el campo educativo (Surange, 2015).

Es imperativo que las organizaciones supervisen su rendimiento en función de las medidas de rendimiento apropiadas y diseñen estrategias para mejoras continuas (Gupta et al., 2019). Investigadores (Visbal-Cadavid et al., 2017) utilizan el Análisis Envolvente de Datos (DEA) para evaluar el desempeño de universidades y adicionalmente utilizan otra etapa para desarrollar procesos de pronósticos (Visbal-Cadavid et al., 2019). Así mismo otros investigadores plantean la importancia de utilizar el análisis envolvente de datos para determinar los niveles de eficiencia en instituciones de educación superior y de esta forma tomar decisiones para mejorar las ineficientes. El análisis de variables claves para evaluar el desempeño de eficiencia en las instituciones de educación superior ha sido abordado utilizando variables como calidad, acreditación de la educación superior, logros y recursos (Visbal-Cadavid et al., 2020)

\section{METODOLOGÍA}

El tipo de investigación es evaluativa, y en un segundo nivel racional, toda vez que inicialmente se identificaron las dimensiones de calidad del servicio analizado, a las cuales se les valoró el desempeño por medio de las métricas de Seis Sigma, específicamente el nivel sigma, para luego proponer de forma racional una estructura DEA que permitió valorar el nivel de eficiencia de los niveles sigma de las dimensiones de calidad del servicio en los 11 periodos objeto de esta investigación. Para lo anterior se realizó un análisis racional propositivo, en donde se integraron la valoración del nivel sigma en las dimensiones de calidad y los conceptos de análisis envolvente de datos, para valor el desempeño de la eficiencia de los niveles sigma de las dimensiones de calidad, buscando la optimización de la dimensión de calidad de respuesta, organización del resultado.

La población utilizada fue el personal especializado de la institución, relacionado con el proceso de gerencia del servicio y las dimensiones establecidas en la Figura 1. Como fuente de información se utilizó la totalidad de registros del centro de atención en donde reposan las estadísticas asociadas a los 11 periodos del 2019 relacionados con las dimensiones de la calidad, garantizando la validez y confiabilidad de la investigación.

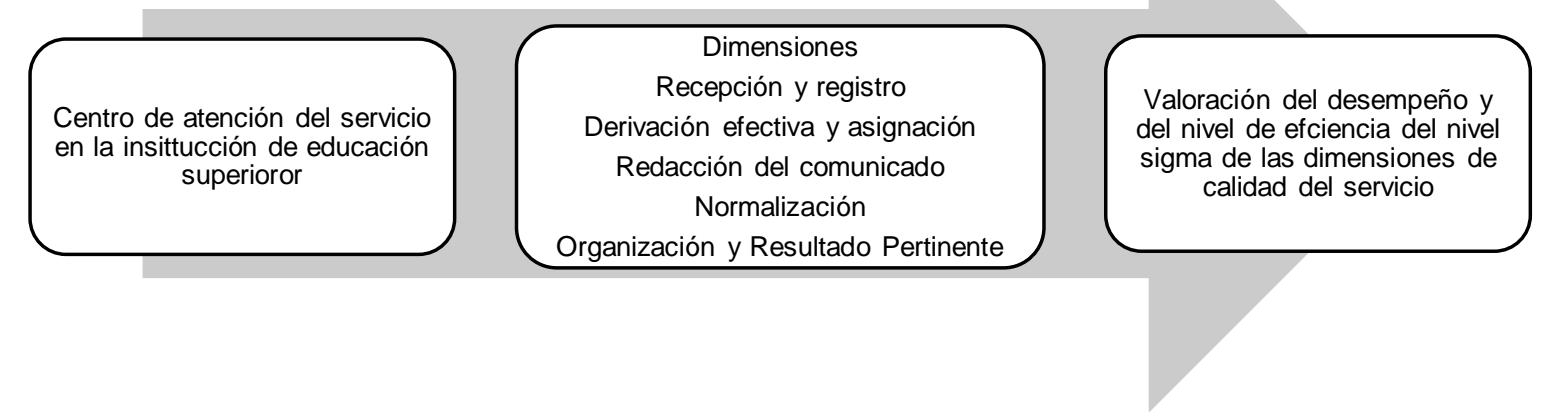

Fig.1: Enfoque valoración del nivel de eficiencia del nivel sigma de las dimensiones de calidad del servicio

Se desarrolló la revisión documental y se contó con los registros propios del servicio prestado asociado con las dimensiones de calidad del servicio evaluado. Para proponer la concepción racional de valoración de las dimensiones de calidad del servicio, se requirió revisar los procesos asociados al objeto de estudio de la investigación. Para lo cual, se desarrollaron entrevistas no estructuradas, al personal responsable del proceso. Para el cálculo de los niveles de eficiencia del nivel sigma se propuso la estructura DEA de entradas y salidas como se muestra en la Tabla 1. Esta se elaboró por medio de un análisis racional para identificar los niveles sigma de las dimensiones de calidad, que se constituyeron en entradas, la variable de salida fue la dimensión de Organización y resultado pertinente. Lo anterior se corrió con la Técnica DEA por medio del software R. 
Tabla 1: Dimensiones de calidad de entrada y salida del modelo DEA establecido

\begin{tabular}{|l|l|}
\hline Dimensiones de calidad de entrada & Dimensiones de calidad de salida \\
\hline Recepción y registro, & Organización y Resultado Pertinente \\
Derivación efectiva y asignación & \\
Redacción del comunicado & \\
Normalización & \\
\hline
\end{tabular}

Para alcanzar los objetivos de esta investigación se propuso un método para evaluar el desempeño y analizar la eficiencia del nivel sigma de las dimensiones de calidad del servicio en una institución de educación superior, en los periodos objeto de esta investigación, que se presenta en la Figura 2.

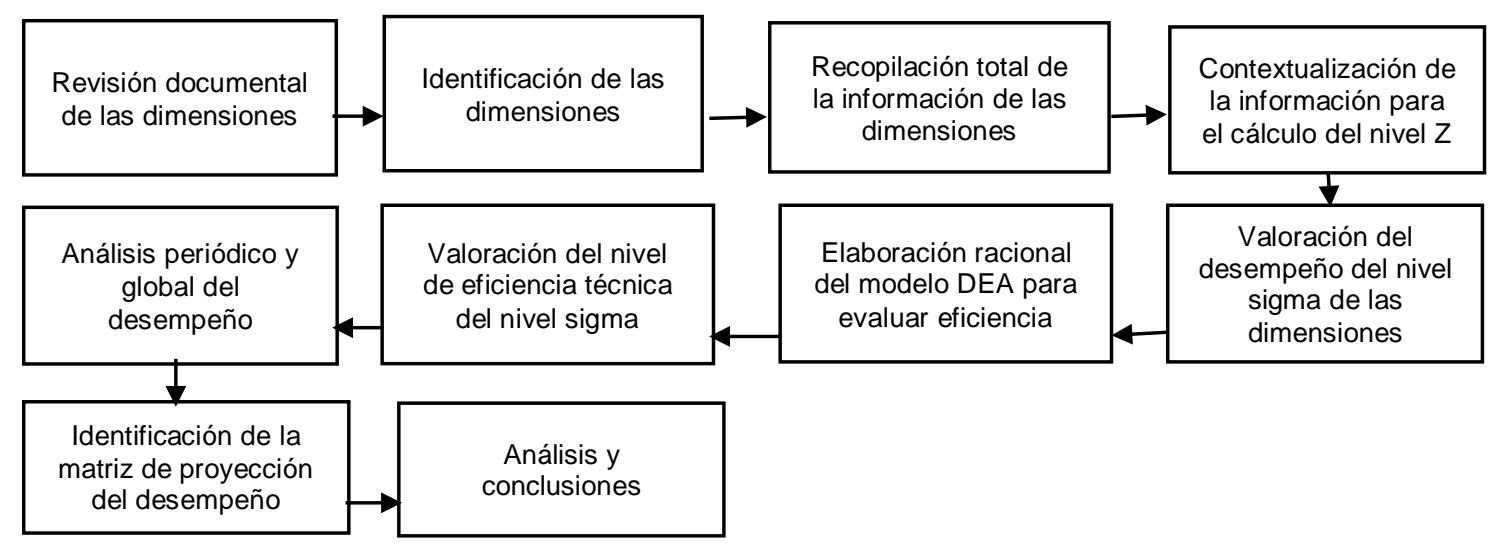

Fig. 2: Método para valorar el desempeño y del nivel de eficiencia de los niveles sigma de las dimensiones de calidad

\section{RESULTADOS}

A continuación, se presentan los datos asociados a las dimensiones de calidad del servicio durante 11 periodos del 2019, los cuales se presentan en la Tabla 2.

Tabla 2: Desempeño de las dimensiones de calidad del servicio

\begin{tabular}{|l|c|c|c|c|c|c|}
\hline Periodo & Resultado & $\begin{array}{c}\text { Recepción } y \\
\text { registro }(O=5)\end{array}$ & $\begin{array}{c}\text { Derivación } \\
\text { efectiva }(O=4)\end{array}$ & $\begin{array}{c}\text { Redacción del } \\
\text { comunicado } \\
(O=5)\end{array}$ & $\begin{array}{c}\text { Organización y } \\
\text { Resultado } \\
(O=3)\end{array}$ & $\begin{array}{c}\text { Normalización } \\
(O=2)\end{array}$ \\
\hline Enero & $U$ & 1356 & 187 & 369 & 494 & 449 \\
& $n$ & 131 & 29 & 27 & 54 & 186 \\
\hline Febrero & $U$ & 2289 & 570 & 501 & 1440 & 1795 \\
& $n$ & 209 & 29 & 75 & 107 & 117 \\
\hline Marzo & $U$ & 3474 & 446 & 635 & 1482 & 1221 \\
& $n$ & 270 & 29 & 24 & 57 & 45 \\
\hline Abril & $U$ & 5842 & 1393 & 1494 & 3420 & 2886 \\
& $n$ & 307 & 253 & 54 & 155 & 334 \\
\hline Mayo & $U$ & 17668 & 1472 & 3387 & 5675 & 5046 \\
& $n$ & 891 & 37 & 95 & 235 & 560 \\
\hline Junio & $U$ & 14447 & 14750 & 3305 & 5742 & 5568 \\
& $n$ & 1150 & 1 & 55 & 556 & 470 \\
\hline Julio & $U$ & 10413 & 1832 & 3539 & 6956 & 3872 \\
& $n$ & 1750 & 51 & 42 & 744 & 298 \\
\hline Agosto & $U$ & 10782 & 1352 & 2359 & 4271 & 2152 \\
& $n$ & 2148 & 26 & 66 & 1650 & 162 \\
\hline Septiembre & $U$ & 10806 & 1488 & 1953 & 4678 & 1840 \\
& $n$ & 1673 & 17 & 27 & 1413 & 79 \\
\hline Octubre & $U$ & 7978 & 1665 & 2075 & 4376 & 2678 \\
& $n$ & 1759 & 32 & 8 & 1201 & 171 \\
\hline Noviembre & $U$ & 9584 & 1576 & 1912 & 5594 & 2143 \\
& $n$ & 1568 & 31 & 3 & 889 & 86 \\
\hline
\end{tabular}


Con base en la información de la Tabla 2 se consolidó la información para la evaluación del servicio. Las fórmulas para calcular las métricas de Seis sigma, Defectos en Partes por Millón de Oportunidades (DPMO) y el nivel Sigma (Z), se presentan a continuación.

$$
\begin{aligned}
& D P M O=\frac{n}{t} \times 1.000 .000=\frac{n}{U x O} \times 1.000 .000 \\
& Z=\sqrt{ }(29.37-2.221 \times \ln (\text { DPMO }))+0.8406
\end{aligned}
$$

Los resultados de las métricas Seis sigma se pueden observar en la Tabla 3, identificando la dimensión de Normalización como la de menor desempeño promedio. Consecuentemente, se procedió a calcular el nivel de eficiencia del nivel sigma de la variable de salida Organización y resultado pertinente, por medio del modelo de Análisis Envolvente de Datos DEA, teniendo como variables de entrada Recepción y registro, Derivación efectiva, redacción del comunicado y la normalización, como se observa en la Tabla 1. Una vez establecido la estructura DEA, considerando las variables de entrada y la variable de salida se utilizó el método de optimización de la variable de salida para obtener el nivel de eficiencia del nivel sigma de la dimensión de calidad Organización y Resultado. Para esto se corrió el modelo DEA a los datos referentes al nivel sigma de las dimensiones de calidad en el software $\mathrm{R}$ con la información presentada en la Tabla 3.

Tabla 3: Resultado de las métricas Nivel sigma y DPMO para las dimensiones de calidad del servicio

\begin{tabular}{|l|c|c|c|c|c|c|}
\hline \multirow{2}{*}{ DMU } & $\begin{array}{c}\text { Métricas Seis } \\
\text { sigma }\end{array}$ & $\begin{array}{c}\text { Recepción } \\
\text { Registro }\end{array}$ & $\begin{array}{c}\text { Derivación y } \\
\text { Asignación }\end{array}$ & $\begin{array}{c}\text { Redacción del } \\
\text { comunicado }\end{array}$ & Normalización & $\begin{array}{c}\text { Organización y } \\
\text { Resultado }\end{array}$ \\
\hline \multirow{2}{*}{ Enero } & Nivel Sigma & 3,57 & 3,27 & 3,68 & 2,32 & 3,3 \\
& DPMO & 19336,28 & 38637,46 & 14652,01 & 207698,5 & 36474,16 \\
\hline \multirow{2}{*}{ Febrero } & Nivel Sigma & 3,59 & 3,74 & 3,39 & 3,35 & 3,47 \\
& DPMO & 18244,73 & 12632,91 & 29801,01 & 32726,6 & 24742,27 \\
\hline \multirow{2}{*}{ Marzo } & Nivel Sigma & 3,66 & 3,64 & 3,92 & 3,59 & 3,73 \\
& DPMO & 15525,33 & 16147,48 & 7653,06 & 18585,4 & 12849,58 \\
\hline \multirow{2}{*}{ Abril } & Nivel Sigma & 3,81 & 3,2 & 3,94 & 3,08 & 3,67 \\
& DPMO & 10525,07 & 45345,91 & 7236,95 & 57842,65 & 15105,265 \\
\hline \multirow{2}{*}{ Mayo } & Nivel Sigma & 3,82 & 3,99 & 4,03 & 3,1 & 3,7 \\
& DPMO & 10086,07 & 6290,49 & 5605,61 & 55488,17 & 13801,22 \\
\hline \multirow{2}{*}{ Junio } & Nivel Sigma & 3,65 & 5,65 & 4,21 & 3,23 & 3,35 \\
& DPMO & 15916,62 & 16,95 & 3355,12 & 42177.67 & 32268,46 \\
\hline \multirow{2}{*}{ Julio } & Nivel Sigma & 3,34 & 3,96 & 4,32 & 3,28 & 3,31 \\
& DPMO & 33617,98 & 6941,03 & 2359,88 & 38427,27 & 35674.24 \\
\hline \multirow{2}{*}{ Agosto } & Nivel Sigma & 3,26 & 4,09 & 4,03 & 3,28 & 2,64 \\
& DPMO & 39848,86 & 4772,48 & 5614,89 & 37639,06 & 128795.9 \\
\hline \multirow{2}{*}{ Septiembre } & Nivel Sigma & 3,37 & 4,26 & 4,27 & 3,53 & 2,79 \\
& DPMO & 30964,04 & 2843,32 & 2771,63 & 21520,8 & 10688,3 \\
\hline \multirow{2}{*}{ Octubre } & Nivel Sigma & 3,21 & 4,08 & 4,66 & 3,36 & 2,84 \\
& DPMO & 44099,49 & 4866,27 & 789,52 & 31925,26 & 91455,55 \\
\hline \multirow{2}{*}{ Noviembre } & Nivel Sigma & 3,35 & 4,08 & 4,9 & 3,56 & 3,12 \\
& DPMO & 32726.89 & 4855.29 & 332.64 & 19990,5 & 52984.71 \\
\hline
\end{tabular}

Los datos del desempeño de eficiencia por periodo, generados por el modelo DEA se presentan en la Tabla 4. Donde se puede observar que los periodos en que se alcanzó la eficiencia del nivel sigma de las dimensiones de calidad de salida fueron enero, febrero, marzo y abril. Es decir, en estos cuatro periodos se prestó un servicio eficiente, de manera integral, asociado con el cumplimiento de las dimensiones de calidad presentadas en la Figura 1.

Tabla 4: Nivel de eficiencia del nivel sigma de las dimensiones de calidad del servicio

\begin{tabular}{|c|c|c|c|c|c|c|c|c|c|c|c|}
\hline DMU & Enero & Febrero & Marzo & Abril & Mayo & Junio & Julio & Agosto & Septiembre & Octubre & Noviembre \\
\hline Eficiencia & 1 & 1 & 1 & 1 & 0,998 & 0,926 & 0,973 & 0,794 & 0,810 & 0,867 & 0,915 \\
\hline
\end{tabular}

De igual manera a pesar de que en los meses de mayo, junio, julio y noviembre, no se alcanzó la eficiencia del $100 \%$. En estos periodos el nivel de desempeño del servicio fue bueno, considerando que la eficiencia para estos periodos estuvo sobre el $91,4 \%$. Cuando se revisa el promedio de eficiencia promedio del nivel sigma de las dimensiones de calidad del centro de atención al cliente en los 11 periodos, se encuentra que esta alcanzó un 93,48\% de desempeño promedio, lo cual es bueno. Sin embargo, en la Tabla 5 se presentan las dimensiones de calidad que requieren mejorar para alcanzar un $100 \%$ de eficiencia. En la Tabla 5 se 
establece el norte de referencia que permite tomar las acciones necesarias para mejorar el nivel de eficiencia del nivel sigma de las dimensiones de calidad de entrada del centro de atención al cliente. De la Figura 3 y la Tabla 5 se puede observar enero, febrero, marzo y abril como los periodos donde se alcanza la eficiencia de 1 del nivel sigma Z, considerado como variable de salida o respuesta Organización y respuesta. Sin embargo, de junio a noviembre de 2019 se puede observar que el nivel de eficiencia del nivel sigma de las dimensiones de calidad en la prestación del servicio analizado multidimensionalmente, generan oportunidades de mejora. Lo anterior requiere que para estos periodos se tomen acciones y decisiones de mejora en las dimensiones de calidad Derivación asignación, redacción del comunicado y Normalización que contribuyan a incrementar la eficiencia operativa relacionada con la eficiencia del nivel sigma de calidad.

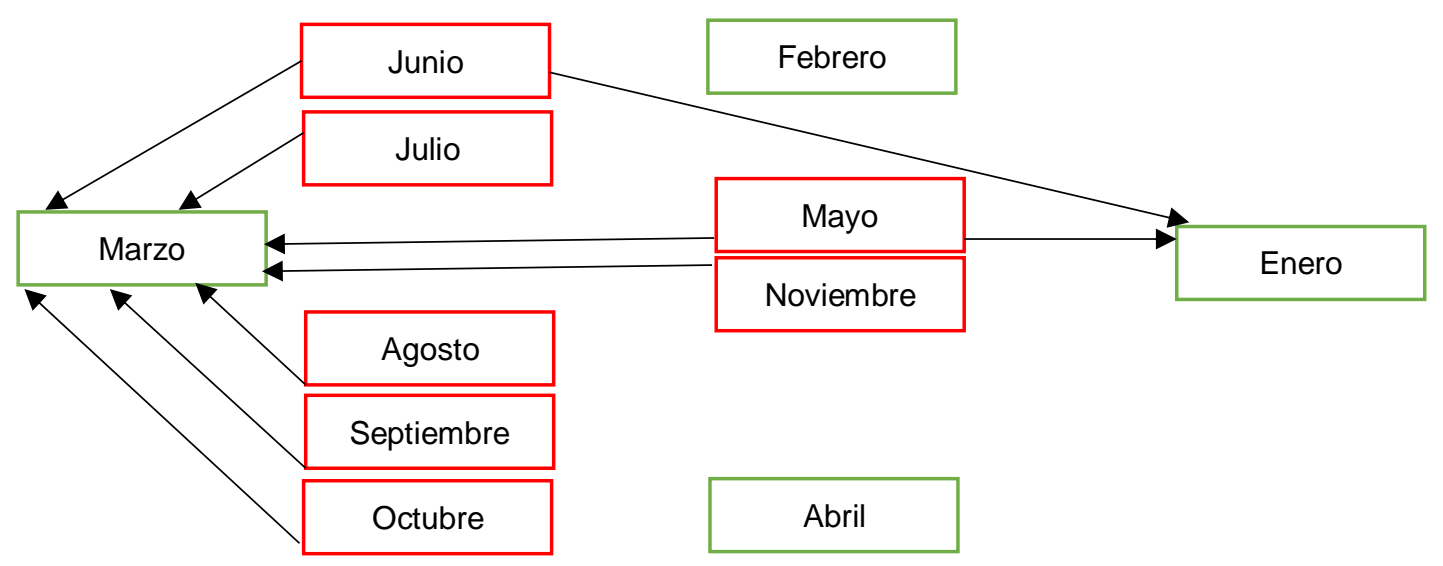

Fig. 3: Meses de referenciación o mejor desempeño del nivel sigma de las dimensiones de calidad.

La Tabla 5 proyecta el incremento en la magnitud requerido del nivel sigma de las dimensiones de calidad de entrada para alcanzar la eficiencia de la dimensión Organización y resultado. Las proyecciones presentadas cuantifican las oportunidades de mejoramiento en términos de nivel sigma requeridos para lograr la eficiencia en la prestación del servicio. Por tanto, este enfoque soporta la toma de decisiones objetivas para planear y controlar actividades de mejoramiento. De la Tabla 5 se puede observar que las variables de Derivación y asignación y redacción del comunicado de mayo a noviembre no tuvieron el mejor desempeño y por tanto se requiere acciones de mejora en estas dos variables. De igual manera la dimensión de Organización y resultado pertinente presenta una oportunidad de mejora en los periodos de julio a noviembre. Lo anterior para garantizar la sostenibilidad de la prestación del servicio y de esta forma llenar las necesidades y expectativas de los usuarios educativo. Por lo anterior, las proyecciones establecidas en la Tabla 5 soportan con evidencia cuantitativa el crecimiento de las magnitudes puntuales del nivel Sigma del servicio, para lograr una eficiencia integral.

Tabla 5: Matriz de proyección de desempeño del nivel sigma de calidad en las dimensiones de calidad de entrada.

\begin{tabular}{|l|c|c|c|c|c|}
\hline Periodo & $\begin{array}{c}\text { Recepción y } \\
\text { registro }\end{array}$ & $\begin{array}{c}\text { Derivación efectiva } \\
\text { y asignación }\end{array}$ & $\begin{array}{c}\text { Redacción del } \\
\text { comunicado }\end{array}$ & $\begin{array}{c}\text { Organización y } \\
\text { Resultado } \\
\text { Pertinente }\end{array}$ & Normalización \\
\hline Enero & 0 & 0 & 0 & 0 & 0 \\
\hline Febrero & 0 & 0 & 0 & 0 & 0 \\
\hline Marzo & 0 & 0 & 0 & 0 & 0 \\
\hline Abril & 0 & 0 & 0 & 0 & 0 \\
\hline Mayo & 0 & 0,341 & 0,014 & 0 & 0 \\
\hline Junio & 0 & 1,94 & 0,313 & 0 & 0 \\
\hline Julio & 0 & 0,621 & 0,724 & 0,005 & 0 \\
\hline Agosto & 0 & 0,672 & 0,428 & 0,072 & 0 \\
\hline Septiembre & 0 & 0,734 & 0,53 & 0,179 & 0 \\
\hline Octubre & 0 & 0,768 & 1,05 & 0,182 & 0 \\
\hline Noviembre & 0 & 0,688 & 1,197 & 0,251 & 0 \\
\hline
\end{tabular}


De igual manera de la Tabla 5 se puede aseverar que la dimensión de calidad recepción y registro y Normalización, no requieren incremento en la dimensión de calidad del servicio, por tanto, se puede aseverar que esta dimensión presentó un buen desempeño en todos los periodos objeto de esta investigación, para lograr un nivel de eficiencia pleno en la variable de respuesta Organización y resultado pertinente. En la Tabla 5 se puede analizar el desempeño real de eficiencia del nivel sigma de la dimensión de calidad, de salida o respuesta. En esta se pueden observar periodo a periodo cuales fueron las dimensiones de calidad que obtuvieron un buen desempeño y cuales tuvieron los desempeños menos favorables para alcanzar la eficiencia de la dimensión de calidad del servicio. Es decir, se tiene una perspectiva del desempeño puntual y global de la eficiencia de las dimensiones de calidad por medio, de la proyección que genera el modelo DEA propuesta para las dimensiones objeto de esta investigación. Lo anterior permite la toma de decisiones sobre las dimensiones de calidad necesarias para lograr una mejora que contribuya con la sostenibilidad en el proceso de prestación del servicio.

\section{DISCUSIÓN}

Los investigadores De la Hoz et al. (2020) han estudiado la gestión de centros de gerencia del servicio, valorando el desempeño de las dimensiones de Calidad del servicio. Sin embargo, este enfoque es limitado frente a lo desarrollado en esta investigación, toda vez que analizan solo el desempeño en dos periodos, mientras que en esta investigación se evalúa el desempeño de forma puntual y longitudinalmente en 11 periodos con la valoración de las métricas de Seis sigma. Y por medio del modelo DEA propuesto y contextualizado a la dimensiones de calidad se evaluó la eficiencia del nivel sigma del servicio, y se obtuvo una valoración global y holística del desempeño del servicio proponiendo un método que articula métricas de Seis sigma y DEA. Es decir, esta investigación aporta siete criterios adicionales para valorar el desempeño. Similar a lo analizado en esta investigación, otros investigadores (Visbal etal., 2020) también han mostrado la pertinencia del análisis de eficiencia para abordar el análisis multicomponente, y analizar el desempeño de las eficiencias en las instituciones de educación superior. En dichos estudios han analizado variables similares como calidad, acreditación, logros, recursos y factores principales, sin embargo, estos estudios tienen la debilidad frente a el modelo planteado que estos valoran el desempeño de manera puntual, mientras que en esta investigación se aporta una matriz de proyección de desempeño del nivel sigma de calidad requerido de las dimensiones de calidad concreta y operativa, que aporta valoraciones para mejorar el servicio objetivamente.

Por lo tanto, la matriz de proyección se constituye en un tablero de control para tomar decisiones bajo evidencia objetiva tal, como lo plantea la filosofía del Seis sigma. De igual manera Visbal-Cadavid et al. (2020) ha utilizado el análisis multifactorial para analizar la calidad universitaria utilizando variables como la acreditación, los logros y los recursos, evidenciando la pertinencia del análisis multivariable para medir el desempeño en la educación superior. Por otro lado, otros trabajos investigativos con un enfoque más global muestran que la calidad en la prestación de los servicios en la educación requiere que se aborden diferentes dimensiones y variables para garantizar la sostenibilidad en la prestación de los servicios (Didham y Ofei, 2018).

\section{CONCLUSIONES}

Como conclusiones de esta investigación se pueden señalar las siguientes:1-. Como contribución única de esta investigación se aporta un método de evaluación del desempeño y análisis de eficiencia del nivel sigma en la calidad del servicio, El cual permite valorar las dimensiones de calidad de calidad del servicio de forma puntual, periódica y longitudinalmente, por medio de las métricas de Seis sigma DPMO y Nivel Sigma. De igual forma se estableció una estructura DEA que permitió valorar el nivel de eficiencia del nivel sigma del servicio de forma global y holística, teniendo como dimensión de salida la optimizada la Organización del resultado, del servicio prestado en la institución de educación; 2-. Como contribución teórica novedosa se articulan los conceptos de calidad del servicio, dimensiones de calidad de un servicio, métricas de Seis sigma, las cuales se contextualizan con las dimensiones de calidad del servicio. Pero lo más significativo y nuevo es la integración racional propositiva entre las métricas de seis sigma y el modelo DEA para valorar el nivel de eficiencia del nivel sigma de la dimensión de calidad Organización y resultado; y 3-Como contribución practica y operativa se propone la Matriz de proyección de desempeño del nivel sigma de calidad requerido de las dimensiones de calidad de entrada, la cual aporta criterios para la toma de decisiones del personal directivo, de tal manera que esta genera las proyecciones necesarias para lograr que el nivel sigma de las dimensiones de calidad de entrada mejoren, para alcanzar la eficiencia técnica de la dimensión de calidad de salida. Es decir, se cuantifica por medio de la proyección generada por el modelo DEA, el incremento de mejora requerido de las dimensiones de calidad de entrada para lograr que la dimensión de calidad salida alcance la eficiencia en la prestación del servicio según el modelo propuesto. 


\section{NOTACIÓN}

\section{Símbolos}

$\mathrm{U}=$ Cantidad de servicios prestados

$\mathrm{O}=$ Oportunidad del error

$\mathrm{n}=$ Número de servicios no conformes

$\mathrm{t}=$ Unidad de tiempo

$\mathrm{Y}=$ Rendimiento del proceso

\section{REFERENCIAS}

Anthony, S. y Antony J., Lean Six Sigma in Academic Institutions-UK vs. Rest of the World, Fourth International Conference on Lean Six Sigma for Higher Education, 68-82 (2017).

Boon, T., Shukur M., Ahmad F., Ahmad N., Evaluation of Service Quality of Private Higher Education Using Service Improvement Matrix, Social and Behavioral Sciences, 224,132-140 (2016).

Capaldo, G., Costantino N., Pellegrino R., The Effect of More Demanding Public Services Quality Standards on the Organization of Service Providers, https://doi.org/10.1080/01900692.2017.1295263, International Journal of Public Administration, 40(10), 847-859 (2017).

De la Hoz, E., Fontalvo T. y Fontalvo O. Evaluación de la calidad del servicio por medio de seis sigma en un centro de atención documental en una Universidad, http://dx.doi.org/10.4067/S0718-50062020000200093, Formación Universitaria, 13 (2), 93-102 (2020).

Didham, R.J y Ofei-Manu P., Advancing policy to achieve quality education for sustainable development. In Issues and trends in Education for Sustainable Development; Leicht, A., Heiss, J., Byun and, W.J., Eds.; United Nations Educational, Scientific and Cultural Organization: Paris, France, (2018).

Fazli, I. y Muzamil M., Exploring competitive priorities in the service sector: evidence from India, https://doi.org/10.1108/IJQSS-02-2018-0021, International Journal of Quality and Service Sciences, 11(2), 167-186 (2019).

Fontalvo, T. y De La Hoz E., Diseño e Implementación de un Sistema de Gestión de la Calidad ISO 9001: 2015 en una Universidad Colombiana, Formación Universitaria, 11(1), 35-44 (2018).

Fontalvo, T., De la Hoz E. y De la Hoz E., Método Análisis Envolvente de Datos y Redes Neuronales en la Evaluación y Predicción de la Eficiencia Técnica de Pequeñas Empresas Exportadoras, Información tecnológica, 29 (6), $267-276$ (2018).

Fontalvo, T., De la hoz E. y Olivos S., Methodology of Data Envelopment Analysis (DEA) - GLMNET for Assessment and Forecasting of Financial Efficiency in a Free Trade Zone - Colombia, Información Tecnológica, 30(5), 263-270 (2019).

Gupta, A., Pachar N., Jha P. C. y Darbari J. D., Efficiency assessment of Indian electronics retail stores using DEA, , https://doi.org/10.1504/IJBPSCM.2019.105719, International Journal of Business Performance and Supply Chain Modelling, 10(4), 386-414 (2019).

Javed, S. y llyas F., Service quality and satisfaction in healthcare sector of Pakistan- the patients' expectations, https://doi.org/10.1108/IJHCQA-08-2016-0110, International Journal of Health Care Quality Assurance, 31(6), 489-501 (2018).

Jiju, A., Lean Six Sigma for higher education, Int. Journal of Productivity and Performance Management, 66(5) (2017).

Shokri, A., Six Sigma in Supply Chain, Supply Chain Strategies, Issues and Models, 63-98 (2014).

Subbarayalu, A. V. y Kuwaiti A., Development of a six sigma rating scale for measuring the quality of work life of teaching staff working in saudi universities, International Journal for Quality Research, 11(2), (2017).

Surange, V., Implementation of Six Sigma to Reduce Cost of Quality: A Case Study of Automobile Sector, Journal of Failure Analysis and Prevention,15(2), 282-294 (2015).

Tumino, M.C. y Poitevin E.R., Evaluación de la calidad de servicio universitario desde la percepción de estudiantes y docentes: caso de estudio, REICE. Revista Iberoamericana sobre Calidad, Eficacia y Cambio en Educación, 12(2), 63-84 (2014).

Visbal-Cadavid, D., Martínez-Gómez M, Escorcia-Cab R., Exploring University Performance through Multiple Factor Analysis: A Case Study, doi:10.3390/su12030924, Sustainability, 12 (3), 924 (2020).

Visbal-Cadavid, D., Martínez-Gómez M. y Guijarro F., Assessing the efficiency of public universities through DEA. A case study, Sustainability, 9(8), 1416 (2017).

Visbal-Cadavid, D., Mendoza A. M., Hoyos I. Q., Prediction of efficiency in colombian higher education institutions with data envelopment analysis and neural networks, https://doi.org/10.1590/0101-7438.2019.039.02.0261, Pesquisa Operacional, 39(2), 261-275 (2019).

Yildiz S.M. y Kara A., Developing Alternative Measures for Service Quality in Higher Education: Empirical Evidence from the School of Physical Education and Sports Sciences, Proceedings of the 2009 Academy of Marketing Science (AMS) Annual Conference, 185-185 (2015). 